\title{
Pregnancy after transcervical radiofrequency ablation guided by intrauterine sonography: case report
}

\author{
José Gerardo Garza-Leal • Iván Hernández León • \\ David Toub
}

Received: 25 October 2013 / Accepted: 25 December 2013 /Published online: 11 January 2014

(C) Springer-Verlag Berlin Heidelberg 2014

\begin{abstract}
Uterine fibroids are a prevalent disorder; with the exception of myomectomy, there are no treatments that are generally accepted as compatible with future fertility and fecundity. Radiofrequency ablation is a minimally invasive treatment modality for uterine fibroids that results in coagulative necrosis and fibroid volume reduction. There have been few reports of pregnancy after laparoscopic and transvaginal radiofrequency ablation of fibroids and no previous reports after a transcervical approach. We report the outcome of the first viable pregnancy after intrauterine sonography-guided radiofrequency ablation of a uterine fibroid.
\end{abstract}

Keywords Fibroids $\cdot$ Radiofrequency ablation $\cdot$ VizAblate

Uterine fibroids are prevalent and often treated with hysterectomy. While several alternatives to hysterectomy exist, over 200,000 hysterectomies are performed for fibroids each year in the USA $[1,2]$. Among alternatives to hysterectomy, only myomectomy is generally considered to be appropriate for women who desire future childbearing. The (ACOG) has advised that uterine artery embolization (UAE) "should be

\footnotetext{
J. G. Garza-Leal • I. H. León

Universidad Autónoma de Nuevo León, Monterrey, Nuevo Leon, Mexico

D. Toub $(\bowtie)$

Gynesonics, Inc., 604 Fifth Avenue, Suite D, Redwood City, CA 94063, USA

e-mail: dtoub@gynesonics.com

D. Toub

Albert Einstein Medical Center, Philadelphia, PA, USA
}

considered investigational or relatively contraindicated in women wishing to retain fertility" [3]. While ACOG has taken note of the reports of successful pregnancies after magnetic resonance imaging-guided focused ultrasound (MRgFUS) therapy [4-8], it maintains that "larger experience is necessary before drawing conclusions" [9].

As with focused ultrasound, radiofrequency energy may be used to thermally ablate fibroid tissue, resulting in coagulative necrosis, fibroid volume reduction, and symptom relief. Few pregnancies have been reported after radiofrequency ablation of uterine fibroids, and none in which the ablation was performed transcervically and under intrauterine sonography guidance. We report the first such viable pregnancy.

\section{Case}

A 41-year-old Gravida 4, Para 2 female presented with abnormal uterine bleeding secondary to uterine fibroids and requested treatment. Transvaginal ultrasonography noted the presence of a midline posterofundal type 1 myoma measuring $1.6 \times 1.6 \mathrm{~cm}$. The patient had previously undergone two Cesarean sections and her medical history was otherwise unremarkable. The patient, who desired uterine preservation, was presented with several options, including enrollment in a clinical trial of transcervical radiofrequency ablation (the FAST-EU trial; Clinicaltrials.gov identifier NCT01226290). The FAST-EU trial is a prospective, nonrandomized, singlearm, multisite trial involving ten sites in Mexico (one site), the UK (five sites), and The Netherlands (four sites) and was approved by the Comisión Federal para la Protección contra Riesgos Sanitarios in Mexico along with the ethics committee of the participating hospital, the Hospital Universitario "Dr. José Eleuterio González" de Universidad Autonoma de Nuevo León in Monterrey, Mexico. The FAST-EU trial has 
been performed in accordance with the ethical standards laid down in the 1964 Declaration of Helsinki and its later amendments.

After a presentation of her care options, the patient elected to be screened for inclusion in the FAST-EU trial. She was using barrier contraception and indicated that she was certain she did not desire future childbearing. The patient met all screening requirements for enrollment and provided her informed consent to participate in the FAST-EU trial and was scheduled for intrauterine sonography-guided, transcervical radiofrequency ablation using the VizAblate ${ }^{\circledR}$ System (Gynesonics, Redwood City, CA, USA).

The VizAblate System uses radiofrequency energy to ablate fibroid tissue and has received CE Marking in the European Union (Fig. 1). The VizAblate treatment device (Fig. 2) is inserted transcervically and includes both an intrauterine sonography probe for visualization and a

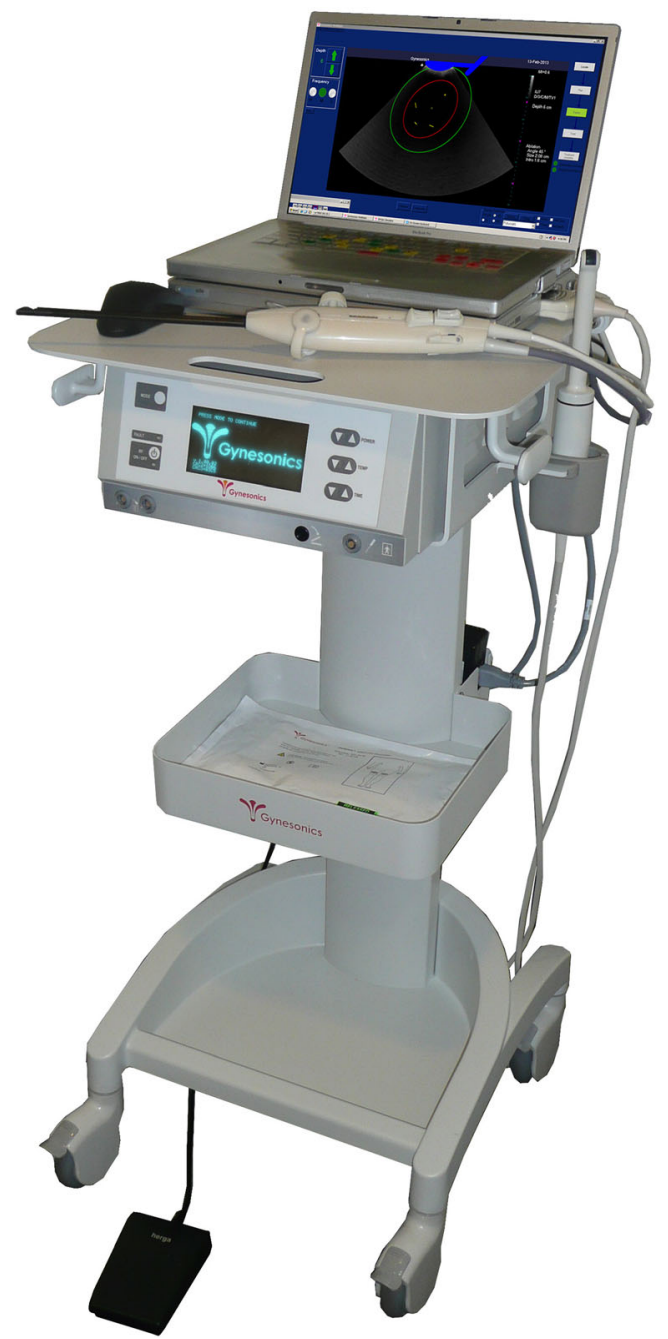

Fig. 1 The VizAblate System. The ultrasound display is provided by the laptop, while the RF generator resides on the second shelf from the bottom radiofrequency needle electrode array for treatment. With a single device, the operator identifies each fibroid using the intrauterine sonography probe and ablates a targeted fibroid with the radiofrequency needle electrode array. The VizAblate System features a graphical interface to display the extent of tissue ablation and heat dissipation, so as to optimize the volume of the fibroid ablation while allowing the gynecologist to avoid thermal injury to the uterine serosa and adjacent structures (Fig. 3). The needle electrode array ablates tissue at a constant $105{ }^{\circ} \mathrm{C}$ for a specific period of time that is a function of the desired ablation volume. The VizAblate System has been described in further detail elsewhere [10].

The patient's baseline menstrual pictogram score was 330 (the minimum requirement for study entry was 120). Her baseline transformed score on the Uterine Fibroid Symptom and Quality of Life (UFS-QOL) Symptom Severity subscale was $53 \%$ and her transformed health-related quality of life subscale score was $2 \%$. While there are no well-defined normal limits for the results of the UFS-QOL questionnaire, Spies, and colleagues originally reported transformed symptom severity and health-related quality of life scores for a normal premenopausal female population as $22.5 \pm 21.1$ and $86.4 \pm 17.7$, respectively [11]. Screening magnetic resonance imaging (MRI) revealed a $1.3-\mathrm{cm}$ midline posterofundal type 1 myoma; there was no suggestion of adenomyosis.

In July, 2012, after a negative pregnancy test, the patient underwent treatment with the VizAblate System under conscious sedation with midazolam and fentanyl. After mechanical cervical dilatation to $25 \mathrm{Fr}$ in the usual fashion, the VizAblate treatment device was introduced into the endometrial cavity. Intrauterine sonography was used to survey the uterus and revealed the type 1 myoma. Using the graphical interface, the ablation location and size were planned by the operating gynecologist so as to maximize the ablation volume of the fibroid while avoiding thermal injury to the uterine serosa. This was accomplished prior to introduction of any device elements within the fibroid. Once the operator was satisfied that the planned ablation was optimal and safe, the distal tip of the VizAblate treatment device was articulated to $45^{\circ}$ and the fibroid was penetrated with a trocar that permitted the needle electrode array to be deployed within the myoma. This was entirely performed under intrauterine sonographic guidance. Rotation of the VizAblate treatment device with concomitant intrauterine sonography in multiple planes confirmed that the uterine serosa was out of harm's way; the planned ablation and its dissipated heat were contained within the uterine serosal margin. A $1.5-\mathrm{cm}$ wide ablation of the targeted fibroid was created with the VizAblate System. This required an ablation duration of $4 \mathrm{~min}$ at a temperature of $105^{\circ} \mathrm{C}$; total procedure time was $16 \mathrm{~min}$. The patient tolerated the procedure well and was discharged that same day. There were no complications reported during or after radiofrequency ablation. 
Fig. 2 The VizAblate Treatment Device
Treatment Planning Control Knob

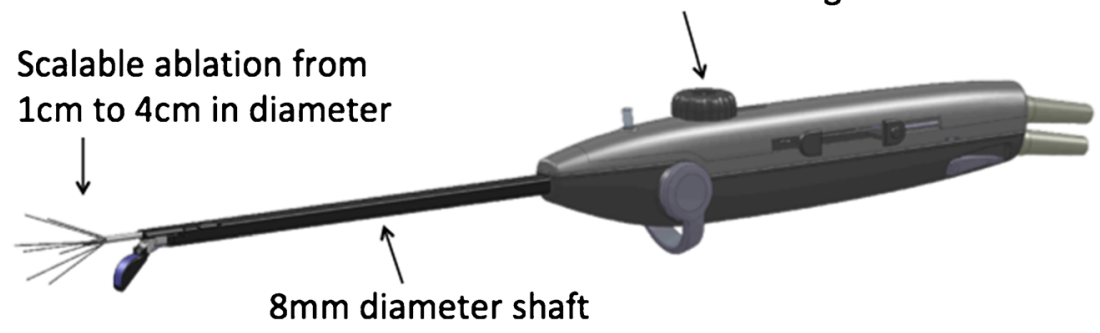

Contrast-enhanced MRI at baseline demonstrated that the total and perfused fibroid volumes were each $1.2 \mathrm{cc}$, indicating $100 \%$ perfusion of the fibroid. At 3 months, both were reduced to $0.1 \mathrm{cc}$, so that both total and perfused fibroid volumes were diminished by $91.7 \%$ from baseline (Fig. 4). The maximum fibroid diameter was reduced from 1.3 to $0.7 \mathrm{~cm}$, a $46.2 \%$ reduction. In terms of symptom relief, the patient's menstrual blood loss was reduced by $45 \%$, as reflected by her 3-month menstrual pictogram score; it fell to 181 from her baseline of 330. The Symptom Severity Score subscale of the UFS-QOL questionnaire fell by $64 \%$, from 53 to $19 \%$. There was similarly improvement in the health-related quality of life subscale, rising from 2 to $97 \%$, an increase of $4750 \%$.

On January 30, 2013, the patient returned for 6-month follow-up and noted that she did not complete her 6-month menstrual pictogram or UFS-QOL questionnaire, as she had not had a period since November 5, 2012. A transvaginal sonogram that day revealed a crown-rump length of $6.1 \mathrm{~cm}$, corresponding to a $124 / 7$-week intrauterine pregnancy. This was consistent with her dates by last menstrual period, with an estimated date of confinement of August 12, 2013. The patient desired to continue her pregnancy and was provided with routine prenatal care. Her pregnancy was unremarkable except for the development of gestational diabetes that was controlled with diet.

On July 30, 2013 at 38 1/7 weeks' gestation, because of her two prior Cesarean deliveries, the patient underwent an elective repeat Cesarean section with intrapartum tubal ligation. This resulted in the birth of a live-born male infant weighing 3,150 $\mathrm{g}$ who received Apgar scores of 9 at $1 \mathrm{~min}$ and 10 at $5 \mathrm{~min}$. Both the infant and mother did well, and there were no complications. The operative report did not note any findings related to fibroids or the prior ablation; there was no indication of a preexisting uterine defect.

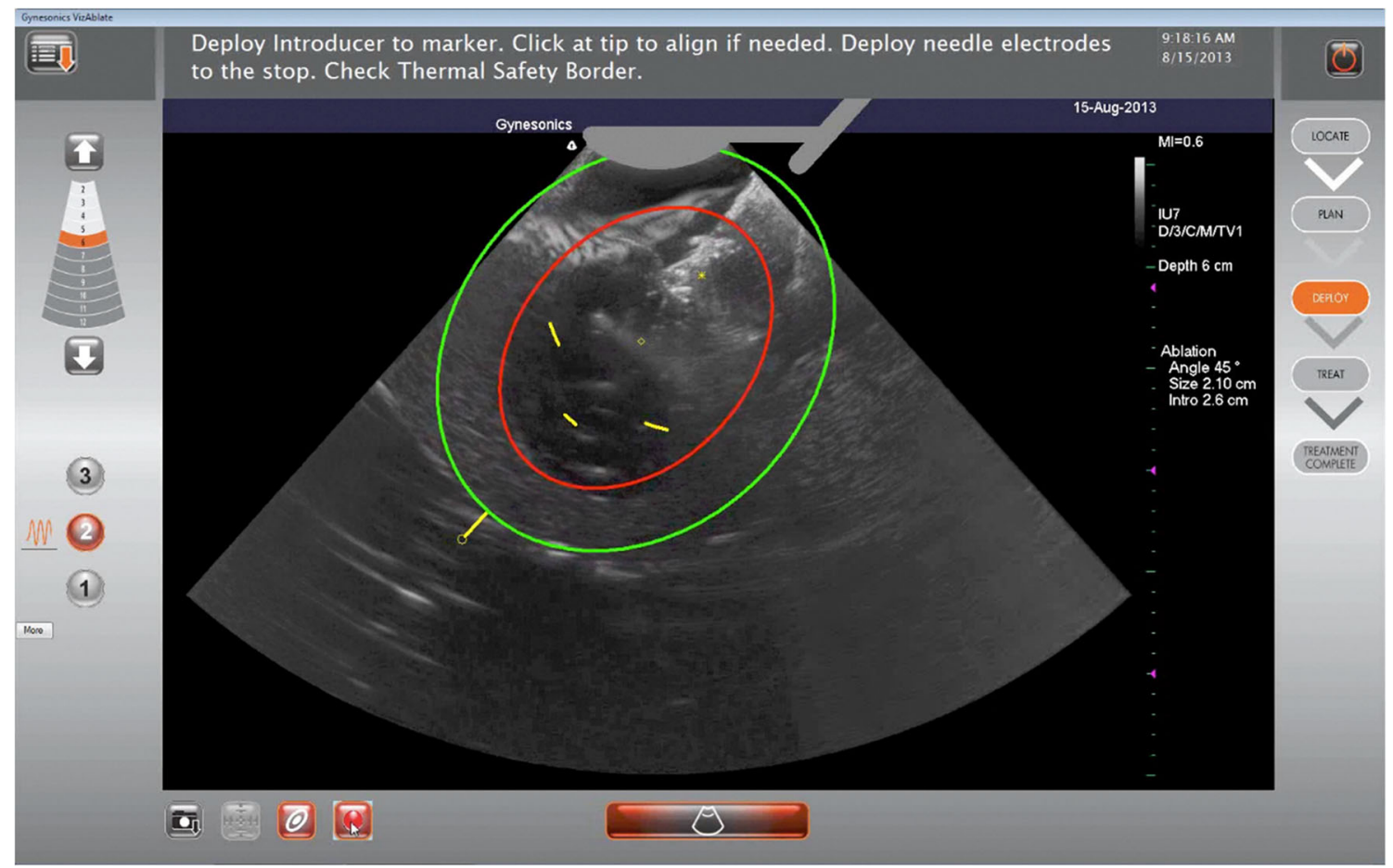

Fig. 3 Intrauterine sonogram of a submucosal fibroid with the VizAblate treatment guides visible (Mean Treatment Region in red and Thermal Safety Border in green). The mean treatment region delineates where the ablation will occur and the thermal safety border demarcates the extent of thermal spread beyond the ablation. The serosa is visible as an echogenic border around the uterus 
Fig. 4 Reduction in total and perfused uterine fibroid volume; baseline, $24 \mathrm{~h}$ and 3-month contrast-enhanced MRI images. Green arrow tip designates the fibroid

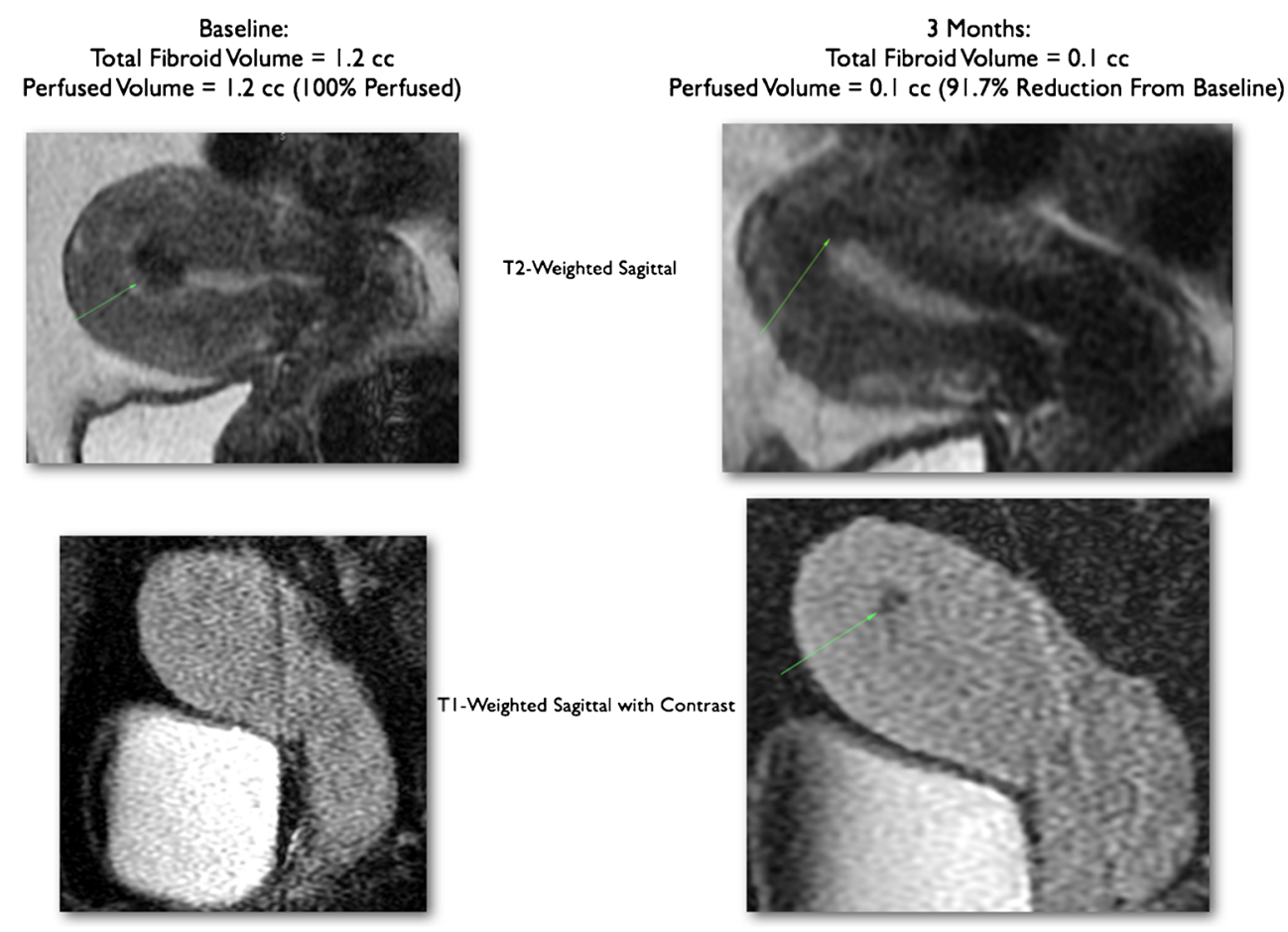

\section{Discussion}

There is interest in a fibroid treatment that is minimally invasive, does not require general anesthesia, and preserves fertility. Hysteroscopic myomectomy is generally considered to preserve fertility, but may be technically challenging and is limited in its ability to treat larger and/or deeper type 1 and type 2 myomata; it is not generally feasible for resection of FIGO types 3,4 , or $2-5$ fibroids. Radiofrequency ablation is an established technology that has been used to effectively treat uterine fibroids [12-20]. Most studies have involved a laparoscopic, percutaneous or transvaginal approach for ablation under laparoscopic, transabdominal or transvaginal sonography. Because the imaging modality is not typically coupled with the radiofrequency treatment device, it can be unwieldy and challenging to perform adequate radiofrequency ablation of fibroids through such approaches. Transcervical radiofrequency ablation, using an intrauterine sonography probe intimately coupled to the treatment device, provides an incisionless approach to treating fibroids. It does not require general anesthesia or uterine distension and can ablate fibroids that include those that are not amenable to hysteroscopic myomectomy. This represents another method by which uterine fibroids may be managed, blending intrauterine sonography for imaging with radiofrequency ablation for treatment.

At present, myomectomy is the only surgical fibroid treatment that is generally considered to be compatible with future pregnancy. Nonetheless, there have been reports describing successful pregnancies after ablative therapies such as MRgFUS and radiofrequency ablation.
In the MRgFUS literature, there have been several reports of successful pregnancies after treatment. Rabinovici and colleagues provided a case report of a 36-year-old woman who underwent MRgFUS for focal adenomyosis [7]. She conceived spontaneously and had an uneventful pregnancy, undergoing a normal spontaneous vaginal delivery at term. There was no evidence of any uterine abnormalities postpartum. In 2008, Rabinovici and his associates also reported the experience with pregnancy after MRgFUS from a prospective registry involving 13 treatment sites in seven nations [6]. There were 54 pregnancies occurring in 51 women, with a mean time to conception of 8 months after MRgFUS. The live birth rate was $41 \%$, with a $28 \%$ spontaneous abortion rate, an $11 \%$ rate of elective abortion, and a $20 \%$ rate of ongoing pregnancies beyond 20 gestational weeks. The mean birth weight was $3.3 \mathrm{~kg}$, and the vaginal delivery rate was $64 \%$. No uterine ruptures were noted. Zaher has also published two separate cases of successful pregnancies after MRgFUS, including one patient who underwent assisted reproduction after her fibroid treatment $[8,21]$.

With regard to pregnancy after contemporary radiofrequency ablation technology, Kim and colleagues treated 69 women with symptomatic fibroids, 13 of whom desired future fertility, using a single-needle radiofrequency electrode connected to a transvaginal sonography probe [20]. Three patients $(4.3 \%)$ conceived and had uncomplicated pregnancies and deliveries (one Cesarean section and two normal spontaneous vaginal deliveries). Again, no uterine ruptures were noted. Berman and colleagues reported their experience in treating a patient with seven fibroids ranging up to $6.1 \mathrm{~cm}$ in diameter via 
laparoscopic radiofrequency ablation with a separate laparoscopic sonography probe for imaging [22]. The patient conceived approximately 3.5 months after treatment and underwent a normal spontaneous vaginal delivery of a 3,487-g infant with Apgar scores of 9 at $1 \mathrm{~min}$ and 9 at $5 \mathrm{~min}$. Post-ablation and post-delivery MRI images indicated a myometrial thickness of $9.6 \mathrm{~mm}$, including beneath the ablation site, suggesting the absence of any myometrial defect.

The experience of our patient adds to this growing literature base relating to thermal ablation of uterine fibroids, particularly via radiofrequency energy. The patient had evidence of symptomatic relief and reductions in both total and perfused fibroid volumes at 3 months, but a longer term assessment was interrupted by her pregnancy. Her pregnancy resulted in a normal outcome via elective repeat Cesarean section.

Many treatment options exist for uterine fibroids, and radiofrequency ablation represents another component in this armamentarium. Additional study must be done to establish the appropriate role of radiofrequency ablation in women who desire future pregnancy. It is not yet established if focal ablation of myomata would typically result in endometrial and/or myometrial injury that would have a negative impact on fertility or fecundity, for example.

Note: Informed consent was obtained from all patients in order to be included in the FAST-EU trial, and this applied to the patient described in this case report.

Conflict of interest Dr. León has no relevant conflicts to disclose. Dr. Garza is a consultant for Gynesonics and has stock options. Dr. Toub is an employee of Gynesonics and has stock options.

\section{References}

1. Day Baird D, Dunson DB, Hill MC et al (2003) High cumulative incidence of uterine leiomyoma in black and white women: ultrasound evidence. Am J Obstet Gynecol 188(1):100-107

2. Dembek CJ, Pelletier EM, Isaacson KB et al (2007) Payer costs in patients undergoing uterine artery embolization, hysterectomy, or myomectomy for treatment of uterine fibroids. J Vasc Interv Radiol 18(10):1207-1213

3. Committee ACOG (2004) Opinion. Uterine artery embolization. Obstet Gynecol 103(2):403-404

4. Gavrilova-Jordan LP, Rose CH, Traynor KD et al (2007) Successful term pregnancy following MR-guided focused ultrasound treatment of uterine leiomyoma. J Perinatol 27(1):59-61

5. Qin J, Chen JY, Zhao WP et al (2012) Outcome of unintended pregnancy after ultrasound-guided high-intensity focused ultrasound ablation of uterine fibroids. Int J Gynaecol Obstet 117(3):273-277
6. Rabinovici J, David M, Fukunishi H et al (2010) Pregnancy outcome after magnetic resonance-guided focused ultrasound surgery (MRgFUS) for conservative treatment of uterine fibroids. Fertil Steril 93(1):199-209

7. Rabinovici J, Inbar Y, Eylon SC et al (2006) Pregnancy and live birth after focused ultrasound surgery for symptomatic focal adenomyosis: a case report. Hum Reprod 21(5):1255-1259

8. Zaher S, Lyons D, Regan L (2011) Successful in vitro fertilization pregnancy following magnetic resonance-guided focused ultrasound surgery for uterine fibroids. J Obstet Gynaecol Res 37(4): 370-373

9. ACOG practice bulletin. Alternatives to hysterectomy in the management of leiomyomas. Obstet Gynecol 2008;112(2 Pt 1): $387-400$

10. Garza-Leal J, Toub D, León I et al (2011) Transcervical, intrauterine ultrasound-guided radiofrequency ablation of uterine fibroids with the VizAblate System: safety, tolerability, and ablation results in a closed abdomen setting. Gynecol Surg 8(3):327334

11. Spies JB, Coyne K, Guaou Guaou N et al (2002) The UFS-QOL, a new disease-specific symptom and health-related quality of life questionnaire for leiomyomata. Obstet Gynecol 99(2):290-300

12. Banks E, Harris M, Garza-Leal J et al (2012) Prospective 12-month follow-up of menstrual blood loss reduction following 135 consecutive cases of radiofrequency volumetric thermal ablation of symptomatic fibroids. J Minim Invasive Gynecol 19(6):S1

13. Bergamini V, Ghezzi F, Cromi A et al (2005) Laparoscopic radiofrequency thermal ablation: a new approach to symptomatic uterine myomas. Am J Obstet Gynecol 192(3):768-773

14. Carrafiello G, Recaldini C, Fontana F et al (2010) Ultrasound-guided radiofrequency thermal ablation of uterine fibroids: medium-term follow-up. Cardiovasc Intervent Radiol 33(1):113-119

15. Cho HH, Kim JH, Kim MR (2008) Transvaginal radiofrequency thermal ablation: a day-care approach to symptomatic uterine myomas. Aust N Z J Obstet Gynaecol 48(3):296-301

16. Chudnoff SG, Levine DJ, Galen DI et al (2012) Prospective 12month follow-up of quality-of-life improvement following 135 consecutive cases of laparoscopic and ultrasound-guided radiofrequency ablation of fibroids. J Minim Invasive Gynecol 19(6):S45

17. Ghezzi F, Cromi A, Bergamini V et al (2007) Midterm outcome of radiofrequency thermal ablation for symptomatic uterine myomas. Surg Endosc 21(11):2081-2085

18. Guido RS, Macer JA, Abbott K et al (2013) Radiofrequency volumetric thermal ablation of fibroids: a prospective, clinical analysis of two years' outcome from the Halt trial. Health Qual Life Outcomes 11(1): 139

19. Iversen H, Lenz S, Dueholm M (2012) Ultrasound-guided radiofrequency ablation of symptomatic uterine fibroids: short-term evaluation of effect of treatment on quality of life and symptom severity. Ultrasound Obstet Gynecol 40(4):445-451

20. Kim CH, Kim SR, Lee HA et al (2011) Transvaginal ultrasoundguided radiofrequency myolysis for uterine myomas. Hum Reprod 26(3):559-563

21. Zaher S, Lyons D, Regan L (2010) Uncomplicated term vaginal delivery following magnetic resonance-guided focused ultrasound surgery for uterine fibroids. Biomed Imaging Interv J 6(2):e28

22. Berman JM, Puscheck EE, Diamond MP (2012) Full-term vaginal live birth after laparoscopic radiofrequency ablation of a large, symptomatic intramural fibroid: a case report. J Reprod Med 57(3-4):159-163 